ANNALES

POLONICI MATHEMATICI

$55(1991)$

\title{
Continuous transformation groups on spaces
}

\author{
by K. SPALlEK (Bochum)
}

To my parents

\begin{abstract}
A differentiable group is a group in the category of (reduced and nonreduced) differentiable spaces. Special cases are the rationals $\mathbb{Q}$, Lie groups, formal groups over $\mathbb{R}$ or $\mathbb{C}$; in general there is some mixture of those types, the general structure, however, is not yet completely determined. The following gives as a corollary a first essential answer. It is shown, more generally,that a locally compact topological transformation group, operating effectively on a differentiable space $X$ (which satisfies some mild geometric property) is in fact a Lie group and operates differentiably on $X$. Special cases have already been known: $X$ a manifold (Montgomery-Zippin), $X$ a reduced (Kerner) or nonreduced (W. Kaup) complex space. The proof requires some analysis on arbitrary differentiable spaces. There one has for example in general no finitely generated ideals as in the case of complex spaces. As a corollary one obtains: The reduction of a locally compact differentiable group is a Lie group (by different methods also proved by Pasternak-Winiarski). It was already proved before that any differentiable group can be uniquely extended to a smallest locally compact differentiable group (as a dense subgroup). The study of the nonreduced parts of differentiable groups remains to be completed.
\end{abstract}

$N$-differentiable spaces ([3], [28], [29]—reduced or not-generalize in case $N=\omega^{*}$ (i.e. of holomorphic functions) complex spaces (reduced or not), in case $N=\omega$ (i.e. of real analytic functions) real-analytic, semi-analytic, or subanalytic spaces (reduced or not); and in all cases $N=1,2, \ldots, \infty$ (i.e. of functions of class $C^{N}$ ) they generalize manifolds of class $C^{N}$ (by admitting arbitrary singularities), or Whitney spaces ([36], [42]), in particular algebras of formal power series. Such spaces arise for example in a natural way as leaf spaces of foliated manifolds $([14],[35])$, the foliations being induced for example by group operations $([22]-[24])$.

$N$-differentiable groups ([33]) are groups in the category of $N$-differen-

1991 Mathematics Subject Classification: 32K15, 59A40, 58C25, 58D05

Key words and phrases: differentiable spaces, differentiable groups, Lie groups, transformation groups, formal groups. 
tiable spaces. They generalize Lie groups and formal groups. As a special consequence of more general results in this paper we deduce (4.2), that any connected reduced locally compact $N$-differentiable group is a Lie group of class $C^{N}$. Thus, even in the category of reduced spaces a group has at most one differentiable structure (for complex spaces this result is quite obvious). By different methods this theorem was also obtained as a main result by Pasternak-Winiarski in his thesis [19] (Warsaw 1981). See [20] for a short survey of this unpublished work [19]. We have announced our results already in [33], indicating there how to continue the classification of $N$-differentiable groups in terms of Lie groups and of formal groups, which we started in [33] and took up recently again $([36])$. In [36] we show, for example, that the nonreduced parts, which one looses by passing to reductions, lead to formal groups (i.e. groups of formal power series).

The above mentioned result is obtained from more general results on continuous transformation groups operating on spaces. Under mild (and necessary) conditions on the distribution of singularities of spaces we show that locally compact topological transformation groups operating effectively on spaces are in fact Lie groups (Main Theorem 3.4) and that they operate differentiably (Theorem 4.1). To simplify proofs, we in general restrict our considerations to $N=\infty, \omega^{*}$. The category of $\omega$-differentiable spaces can be considered as a full subcategory of $\omega^{*}$-differentiable spaces and is thus covered as well. Also complex spaces form a full subcategory of all $\omega^{*}$ differentiable spaces.

The special cases of reduced spaces generalize the corresponding results for manifolds $([17])$, in particular the case $N=\omega^{*}$ extends the results of [11], [10] for reduced (respectively nonreduced) complex spaces. The new proofs, which are necessary in our general setting, are more involved than in the classical situations (for example: ideals in the case $N=\infty$ are almost never finitely generated). Besides some constructions in the category of $N$-differentiable spaces we need and develop some additional "nonreduced analysis", to which we extend the methods and results of "reduced analysis" from [17], [41]. Together with 4.2 and [33], [36] we show in particular that any $C^{N}$-differentiable group $G(N \geq \infty, G$ reduced or not) can be uniquely "extended" to a "complete" $C^{N}$-differentiable group $\widehat{G}$ such that red $\widehat{G}$ is a Lie group, that $G$ is "dense" in $\widehat{G}$ and that $\widehat{G}$ is somehow a family of formal groups parametrized along the Lie group red $\widehat{G}$. Those parts of our results which deal with reduced groups, were - by different methods - also obtained in [16], [19]-[21]; [16] gives for this special case a simpler proof; however, it should be possible to simplify it even more.

$\S 1$. The category $\mathcal{R}^{l, k}$ of mixed $(l, k)$-differentiable spaces. Mixed spaces of a special type were introduced and used by M. Jurchescu in his 
theory of mixed spaces (for example [9]). We give a short introduction to the more general situations which we need here.

A sheaf $\mathcal{G}$ of vector spaces on a topological space $X$ will be identified with its canonical sheaf datum $\left\{H^{0}(U, \mathcal{G}) \mid U \subset X\right.$ open $\}$. $\mathcal{G}$ is called a topological sheaf if each $H^{0}(U, \mathcal{G})$ is in addition a topological vector space in such a way that the canonical restriction operators $H^{0}(U, \mathcal{G}) \rightarrow H^{0}(V, \mathcal{G})$ for $V \subset U$ are continuous. $\mathcal{G}$ is a Fréchet sheaf if each $H^{0}(U, \mathcal{G})$ is a Fréchet space. Topological spaces are assumed to have countable topology.

Differentiable and mixed differentiable spaces are special cases of ringed spaces. We explain their local models:

Let $K, K^{\prime}$ denote $\mathbb{C}$ if $N=\omega^{*}$ is involved, and $\mathbb{R}$ in all cases $N \neq \omega^{*}$, and let $K$ also denote an arbitrary locally compact topological space in case $N=0$. Write $x=\left(x_{1}, \ldots, x_{n}\right) \in K^{n}$. For $l, k \in\left\{0,1, \ldots, \infty, \omega^{*}\right\}$ let $\mathcal{D}^{k}$ denote the sheaf of germs of functions of class $C^{k}$ on $K^{n}$ (real-valued if $\left.k \neq \omega^{*}\right)$. Let $\mathcal{D}^{l, k}$ denote the sheaf of germs of functions on $K^{n} \times K^{\prime m}$ of mixed class $C^{l, k}$ : For $U \subset K^{n}, V \subset K^{\prime m}$ open a function

$$
f: U \times V \rightarrow K^{\prime \prime}
$$

is of class $C^{l, k}$ iff all those partial derivatives of $f$ exist and are continuous where only derivatives up to order $l$ in the first variable $x$ of $K^{n}$ and up to order $k$ in the second variable $y$ of $K^{\prime m}$ appear. Here $K^{\prime \prime}=\mathbb{C}$ iff at least one of $l, k$ is $\omega^{*}$. And if for example $l=\omega^{*}$, hence $K=\mathbb{C}$, then differentiability up to order $\omega^{*}$ just means holomorphy. By formal reasons let $\mathcal{D}^{-1, k}=\mathcal{D}^{l,-1}=\mathcal{D}^{-1,-1}$ denote the sheaf of zero-functions. For any open $U$ let $H^{0}\left(U, \mathcal{D}^{k}\right)$ carry the topology of uniform convergence on compact subsets of $U$ of sequences in $H^{0}\left(U, \mathcal{D}^{k}\right)$, together with their derivatives up to order $k$ (in case $\left.k \neq 0, \omega^{*}\right)$. With this topology $\mathcal{D}^{k}$ is a Fréchet sheaf. In a similar way $\mathcal{D}^{l, k}$ is a Fréchet sheaf. Each $f \in H^{0}\left(U \times V, \mathcal{D}^{l, k}\right)$ can also be considered as a mapping $U \rightarrow H^{0}\left(V, \mathcal{D}^{k}\right)$ of class $C^{l}$. Note that $\mathcal{D}^{l, k}$ is always a sheaf on a specific decomposition $K^{n} \times K^{\prime m}$ which one has to bear in mind. In case of different $n$ 's, $m$ 's, $K$ 's we may use the same symbol $\mathcal{D}^{l, k}$ or others, for example $\widetilde{\mathcal{D}}^{l, k}$, to distinguish in case of need. We have $\mathcal{D}^{l, l}=\mathcal{D}^{l}$ if $l=0, \infty, \omega^{*}$; also $\mathcal{D}^{l, k}=\mathcal{D}^{l}$ if $m=0$, hence $K^{m}=\{0\}$. Let $l \pm 1=l$ for $l=\infty, \omega^{*}$.

DEFINITION 1.1. (a) An $l$, $k$-differentiable space in $K^{n} \times K^{m}$ is a ringed space $D^{l, k}=\left(D, \mathcal{D}^{l, k} / \mathcal{I}\right) \subset K^{n} \times K^{m}$. Here $D \subset K^{n} \times K^{m}$ is an arbitrary subset and $\mathcal{I} \subset \mathcal{D}^{l, k} \mid D$ is an ideal subsheaf with $\mathcal{I}_{p} \neq \mathcal{D}_{p}^{l, k}$ for each stalk, $p \in D$.

(b) For $W \subset K^{n} \times K^{m}, W^{\prime} \subset K^{s} \times K^{t}$ open, a morphism

$$
\psi=(\underline{\psi}, \underline{\underline{\psi}}):\left(W, \mathcal{D}^{l, k} \mid W\right) \rightarrow\left(W^{\prime}, \widetilde{\mathcal{D}}^{l^{\prime}, k^{\prime}} \mid W^{\prime}\right)
$$


of ringed spaces $\left(\underline{\psi}: W \rightarrow W^{\prime}, \quad \underline{\underline{\psi}}: W \oplus_{\underline{\psi}} \widetilde{\mathcal{D}}^{l^{\prime}, k^{\prime}} \rightarrow \mathcal{D}^{l, k}\right.$, shorter $\underline{\underline{\psi}}:$ $\left.\widetilde{\mathcal{D}}^{l^{\prime}, k^{\prime}} \rightarrow \mathcal{D}^{l, k}\right)$ is called differentiable if $\underline{\underline{\psi}}(f)=f \circ \underline{\underline{\psi}}$ for any $f \in \widetilde{\mathcal{D}}^{l^{\prime}, k^{\prime}}$ and if $\psi$ thus induces continuous mappings of sheaves $\overline{\widetilde{\mathcal{D}}^{l^{\prime}}, k^{\prime}} \rightarrow \mathcal{D}^{l, k}, \widetilde{\mathcal{D}}^{l^{\prime}-1, k^{\prime}} \rightarrow$ $\mathcal{D}^{l-1, k}, \widetilde{\mathcal{D}}^{l^{\prime}, k^{\prime}-1} \rightarrow \mathcal{D}^{l, k-1}$.

(c) A morphism of ringed spaces $\psi: D^{l, k} \rightarrow \widetilde{D}^{l^{\prime}, k^{\prime}}$ is called differentiable if for each $(x, y) \in D$ there exist neighbourhoods $U(x, y) \subset K^{n} \times K^{m}$, $V(\psi(x, y)) \subset K^{s} \times K^{t}$ with differentiable $\bar{\psi}:\left(U, \mathcal{D}^{l, k} \mid U\right) \rightarrow\left(V, \widetilde{\mathcal{D}}^{l^{\prime}, k^{\prime}} \mid V\right)$ so that the following diagram is commutative:

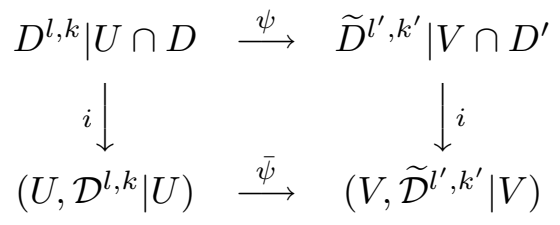

Here $i$ denotes natural embeddings.

(d) $D^{l, k}$ is called reduced if $\mathcal{I}$ is the sheaf of all germs of $\mathcal{D}^{l, k}$ vanishing on $D$. We say that $D^{l, k}$ satisfies

$\mathrm{A}_{1}$ if $D$ is locally compact;

$\mathrm{A}_{2}$ if $\mathcal{I} \subset \mathcal{D}^{l, k} \mid D$ is closed in the topology of $\mathcal{D}^{l, k}$ (see also [32], symbolically: $\mathcal{I}=\overline{\mathcal{I}})$

$\mathrm{A}_{3}$ if $\mathcal{I}=\mathcal{I}\left(\mathcal{I} \cdot \mathcal{D}^{l-1, k}+\mathcal{I} \cdot \mathcal{D}^{l, k-1}\right) \cap \mathcal{D}^{l, k}$ (here $\mathcal{I} \cdot \mathcal{D}{ }^{\prime \prime}$ denotes the sheaf generated by $\mathcal{I}$ over $\mathcal{D}^{\cdot, \cdot)}$;

$\mathrm{A}_{4}$ if $\mathcal{I}=\overline{\left(\mathcal{I} \cdot \mathcal{D}^{l-1, k}\right.}+\overline{\left.\mathcal{I} \cdot \mathcal{D}^{l, k-1}\right)} \cap \mathcal{D}^{l, k}$ (here the bars denote the topological closures in $\mathcal{D}^{l-1, k}$, resp. in $\left.\mathcal{D}^{l, k-1}\right)$;

$\mathrm{A}_{5}$ if $\mathrm{A}_{1}, \mathrm{~A}_{4}$ are satisfied; $D^{l, k}$ is then called a standard space.

It is not important at this place to know precisely all differentiable morphisms. In the following only two cases will appear, where (b) will be quite obvious.

Remark 1.2. The composition of differentiable morphisms is differentiable. In number spaces embedded mixed $(l, k)$-differentiable spaces and their differentiable morphisms form a category. The diffeomorphisms in this category keep invariant each of the properties $\mathrm{A}_{i}$. By "glueing together" embedded spaces we obtain in the category of ringed spaces the subcategories $\mathcal{R}^{l, k}$ of abstract $(l, k)$-differentiable spaces and $\mathcal{R}_{i}^{l, k}$ of spaces, satisfying in "local charts" the property $\mathrm{A}_{i}$. All this is similar to the unmixed cases in [29]. In a natural way we have from [29] for the category $\mathcal{R}^{l}$ of $l$-differentiable spaces (resp. $\mathcal{R}_{i}^{l}$ of those satisfying $\mathrm{A}_{i}$ ): $\mathcal{R}^{l} \subset \mathcal{R}^{l, k}, \mathcal{R}_{i}^{l} \subset \mathcal{R}_{i}^{l, k}$, for any $k$ (see $\S 2$ ). Each point $p$ is in a natural way an $l$-differentiable space $(p, \mathbb{R})$ for $l \neq \omega^{*}\left(\operatorname{resp} .(p, \mathbb{C})\right.$ for $\left.l=\omega^{*}\right)$ and therefore

$$
\mathcal{R}^{k} \ni X=p \underline{\times} X \in \mathcal{R}^{l, k} .
$$


Note. A space $X$ is a pair $(\underline{X}, \underline{\underline{X}})$ with an underlying topological space $\underline{X}$ and a certain sheaf $\underline{\underline{X}}$ on $\underline{X}$.

$\S 2$. Products and pseudoproducts of spaces. We extend the notion of products $\times$ and pseudoproducts $\underline{x}$ for $N$-differentiable spaces from [31], [33] to the case of mixed differentiable spaces. We consider the following data:

$$
\begin{aligned}
D^{l}:= & \left(D, \mathcal{D}^{l} / \mathcal{I}\right) \subset \mathbb{R}^{n}, \quad D^{\prime k}:=\left(D^{\prime}, \mathcal{D}^{k} / \mathcal{I}^{\prime}\right) \subset \mathbb{R}^{m} \\
\mathcal{I}+\mathcal{I}^{\prime}:= & \left(\mathcal{I} \cdot \mathcal{D}^{l-1, k}+\mathcal{I} \cdot \mathcal{D}^{l, k-1}\right. \\
& \left.+\mathcal{I}^{\prime} \cdot \mathcal{D}^{l-1, k}+\mathcal{I}^{\prime} \cdot \mathcal{D}^{l, k-1}\right) \cap \mathcal{D}^{l, k} \mid D \times D^{\prime}
\end{aligned}
$$

$\mathcal{I} \pm \mathcal{I}^{\prime} \subset \mathcal{D}^{l, k} \mid D \times D^{\prime}$ with stalks

$\left(\mathcal{I} \pm \mathcal{I}^{\prime}\right)_{\left(x^{0}, y^{0}\right)}:=\left\{f \in \mathcal{D}_{\left(x^{0}, y^{0}\right)}^{l, k} \mid f\right.$ has a representative

$$
F: U\left(x^{0}\right) \times V\left(y^{0}\right) \rightarrow \mathbb{R} \text { with } F(x,-)_{y} \in \mathcal{I}_{y}^{\prime}, F(-, y)_{x} \in \mathcal{I}_{x}
$$
for each $\left.x \in D \cap U, y \in D^{\prime} \cap V\right\}$,

$$
\left(\mathcal{I} \mp \mathcal{I}^{\prime}\right):=\overline{\mathcal{I}+\mathcal{I}^{\prime}}
$$

In case $l=0$ (respectively $k=0$ ) $D^{0}$ (resp. $D^{\prime 0}$ ) stands as before for an arbitrary given topological space $D$ (resp. $D^{\prime}$ ) with its reduced structure of continuous functions: $\mathcal{I}=0$ (resp. $\left.\mathcal{I}^{\prime}=0\right)$. In case $l=\omega^{*}\left(\right.$ resp. $\left.k=\omega^{*}\right)$ $\mathbb{R}$ stands for $\mathbb{C}$ (hence $\mathbb{R}^{n}$ for $\mathbb{C}^{n}$, resp. $\mathbb{R}^{m}$ for $\mathbb{C}^{m}$ ). Now, set

$$
\begin{aligned}
D^{l} \times D^{\prime k} & :=\left(D \times D^{\prime}, \mathcal{D}^{l, k} / \mathcal{I}+\mathcal{I}^{\prime}\right) & & \text { (product space) } \\
D^{l} \times D^{\prime k} & :=\left(D \times D^{\prime}, \mathcal{D}^{l, k} / \mathcal{I} \pm \mathcal{I}^{\prime}\right) & & \text { (pseudoproduct space) } \\
D^{l} \overline{\times} D^{\prime k} & :=\left(D \times D^{\prime}, \mathcal{D}^{l, k} / \mathcal{I} \mp \mathcal{I}^{\prime}\right) & & \text { (closed product space) }, \\
D^{l} \times{ }_{r} D^{\prime k} & :=\operatorname{red}\left(D^{l} \times D^{\prime k}\right) & & \text { (reduced product space) } .
\end{aligned}
$$

With - denoting one of these products one has the natural projections $\pi_{1}, \pi_{2}: D^{l} \bullet D^{\prime k} \rightarrow\left(D^{l}, D^{\prime k}\right)$, and if $D^{l}, D^{\prime k}$ satisfy $\mathrm{A}_{3}$, the natural embeddings

$$
D^{l} \times_{r} D^{\prime k} \hookrightarrow D^{l} \times D^{\prime k} \hookrightarrow D^{l} \times D^{\prime k}, \quad D^{l} \times D^{\prime k} \hookrightarrow D^{l} \times D^{\prime k} ;
$$

in addition, if also $\mathrm{A}_{2}$ is satisfied,

$$
D^{l} \times D^{\prime k} \hookrightarrow D^{l} \overline{\times} D^{\prime k} .
$$

Additional spaces $D_{\sim}^{l} \subset \mathbb{R}^{n}, D_{\sim}^{\prime k} \subset \mathbb{R}^{t}$ with differentiable morphisms $\varphi$ : $D^{l} \rightarrow D_{\sim}^{l}, \psi: D^{\prime k} \rightarrow D_{\sim}^{\prime k}$ induce in a natural way (see [31] for the cases $l=k$ ) differentiable morphisms

$$
\varphi \bullet \psi: D^{l} \bullet D^{\prime k} \longrightarrow D_{\sim}^{l} \bullet D_{\sim}^{\prime k}, \quad \bullet \in\left\{\times, \underline{\times}, \times_{r}, \overline{\times}\right\},
$$

which are compatible with our projections $\pi_{i}$. Indeed, if $\Phi($ resp. $\Psi$ ) is a local representative of $\varphi$ in $\mathbb{R}^{n}$ (resp. of $\psi$ in $\mathbb{R}^{m}$ ), then $\Phi \times \Psi$ is a local 
representative of $\varphi \bullet \psi$ in $\mathbb{R}^{n} \times \mathbb{R}^{m}$. One shows (as in [31]) that this construction of $\varphi \bullet \psi$ does not depend on the choice of the representatives of $\varphi$ and $\psi$. The mappings $\left(D^{l}, D^{\prime k}\right) \rightsquigarrow D^{l} \bullet D^{\prime k},(\varphi, \psi) \rightsquigarrow \varphi \bullet \psi$, extend now in a natural unique way to mappings $\left(X^{l}, X^{\prime k}\right) \longrightarrow X^{l} \bullet X^{\prime k}$ on not necessarily embedded spaces $X^{l} \bullet X^{\prime k}$, which are functorial on the corresponding categories:

$$
\text { • : }\left(\mathcal{R}^{l}, \mathcal{R}^{k}\right) \rightsquigarrow \mathcal{R}^{l, k} \text {. }
$$

The projections $\pi_{i}$ above also extend to projections

$$
\pi_{1}, \pi_{2}: X^{l} \bullet X^{\prime k} \longrightarrow\left(X^{l}, X^{\prime k}\right) .
$$

If properties $\mathrm{A}_{2}, \mathrm{~A}_{3}$ are satisfied, one has natural embeddings

$$
X^{l} \times_{r} X^{\prime k} \hookrightarrow X^{l} \times X^{\prime k} \hookrightarrow X^{l} \overline{\times} X^{\prime k} \hookrightarrow X^{l} \times X^{\prime k} .
$$

Note 2.1. Let $p$ be a point, considered as a reduced $j$-differentiable space. Then

( $\alpha) p \times X \simeq p \times X \simeq X$, and $p \overline{\times} X \simeq X$ if $X$ is closed.

( $\beta$ ) Let $T$ denote a topological space. Then two morphisms $f, g$ : $T \times X \longrightarrow Y$ are equal iff the compositions

$$
f_{p}: X \longrightarrow p \underline{\times} X \longrightarrow T \underline{\times} X \stackrel{f}{\longrightarrow} Y, \quad g_{p}: X \longrightarrow p \underline{\times} X \longrightarrow T \underline{\times} X \stackrel{g}{\longrightarrow} g
$$

are equal for each $p \in T$.

Also, for the special cases $l=k=\infty, \omega, \omega^{*}$, note that: $\times_{r}$ is the product in the category of reduced spaces; $\bar{x}$ is the product in the category of closed spaces; $\times_{r}=\underline{x}$ in the category of reduced spaces; $\times_{r}=\underline{\times}=\overline{\times}=\times$ in the category of topological spaces or differentiable manifolds.

Fortunately there are still some other identities between the different products in important special cases. To see this we first define:

Definition. An embedded space $D^{l}=\left(D, \mathcal{D}^{l} / \mathcal{I}\right) \subset \mathbb{R}^{n}$ is called locally finitely generated if $\mathcal{I}$ as a sheaf in $\mathcal{D}^{l} \mid D$ is locally finitely generated over $\mathcal{D}^{l} \mid D$. An abstract space $X^{l}$ is locally finitely generated if each of its local models $\mathcal{D}^{l}$ (given by charts) is locally finitely generated. We abbreviate l.f.g. for "locally finitely generated".

Not e. The property "l.f.g." of $D^{l}$ does not depend on a given embedding of $D^{l}$. Therefore $X^{l}$ is already l.f.g. if this holds only for the models of the charts of some atlas of $X^{l}$.

THEOREM 2.2. $(\alpha) X \times{ }_{r} Y=X \times Y$ for reduced spaces $X, Y$.

( $\beta) X \times Y=X \times Y$ if $X$ is a locally compact topological space or a $C^{l}$-manifold and $Y$ is a $k$-differentiable standard space with $k=\infty, \omega, \omega^{*}$ which is l.f.g. (for example: manifold, complex space).

$(\gamma) X \times Y=X \overline{\times} Y$ if $X$ is a $C^{l}$-manifold, $Y$ a closed $C^{l}$-space, $l \in$ $\left\{\infty, \omega, \omega^{*}\right\}$. 
( $\delta) X \underline{\times} Y=X \times Y$ if $X$ is a $C^{l}$-manifold, $Y$ a $C^{l}$-space, $l \in\left\{\omega, \omega^{*}\right\}$.

Proof. $(\alpha)$ is obvious.

$(\beta)$ is of local nature. We therefore may assume: $X=D^{l}=\left(D, \mathcal{D}^{l}\right), Y=$ $D^{\prime k}=\left(D^{\prime}, \mathcal{D}^{\prime k} / \mathcal{I}^{\prime}\right) \subset \mathbb{R}^{m}$. Hence $0+\mathcal{I}^{\prime}=\mathcal{I}^{\prime} \cdot \mathcal{D}^{l, k}$, and $f \in\left(0 \pm \mathcal{I}^{\prime}\right)_{\left(x^{0}, y^{0}\right)} \Leftrightarrow$ $f$ has a representative $F \in H^{0}\left(U\left(x^{0}\right) \times V\left(y^{0}\right), \mathcal{D}^{l, k}\right)$ with $\left.\overline{F(x,-}\right)_{y} \in \mathcal{I}_{y}^{\prime}$, $F(-, y)_{x} \equiv 0, \forall x \in U\left(x^{0}\right) \cap D, y \in V\left(y^{0}\right) \cap D^{\prime} \Leftrightarrow f$ has a representative $F \in H^{0}\left(U \times V, \mathcal{D}^{l, k}\right)$ with $F(x,-)_{y} \in \mathcal{I}_{y}^{\prime}, \forall x \in U, y \in V \cap D^{\prime} \Leftrightarrow f$ has a representative $F \in H^{0}\left(U \times V, \mathcal{D}^{l, k}\right)$ which can be considered as a $C^{l}$ differentiable mapping $U \rightarrow H^{0}\left(V, \mathcal{I}^{\prime}\right)$ (here, without loss of generality $\mathcal{I}^{\prime}$ is considered as a locally finitely generated closed sheaf on $V) \Leftrightarrow f$ has a representative $F \in H^{0}\left(U, \mathcal{D}^{l}\right) \widehat{\otimes}_{\varepsilon} H^{0}\left(V, \mathcal{I}^{\prime}\right)$ (see [41], part III). Since $\mathcal{I}^{\prime}$ is l.f.g. we have for a small enough neighbourhood $V=V\left(y^{0}\right) \subset \mathbb{R}^{m}$ a surjection $H^{0}\left(V, \mathcal{D}^{\prime k}\right)^{s} \rightarrow H^{0}\left(V, \mathcal{I}^{\prime}\right)$. Then the following induced mapping is also surjective:

$H^{0}\left(U \times V, \mathcal{D}^{l, k}\right)^{s} \rightarrow\left(H^{0}\left(U, \mathcal{D}^{l}\right) \widehat{\otimes}_{\varepsilon} H^{0}\left(V, \mathcal{D}^{\prime k}\right)\right)^{s} \rightarrow H^{0}\left(U, \mathcal{D}^{l}\right) \widehat{\otimes}_{\varepsilon} H^{0}\left(V, \mathcal{I}^{\prime}\right)$.

This implies, however, $0+\mathcal{I}^{\prime}=0 \pm \mathcal{I}^{\prime}$, hence $(\beta)$.

$(\gamma)$ Let first $l=\infty$. Assume, without loss of generality, $X=U \subset \mathbb{R}^{r}$ open, $Y=D^{\prime l}=\left(D^{\prime}, \mathcal{D}^{\prime l} / \mathcal{I}^{\prime}\right) \subset \mathbb{R}^{m}, \mathcal{I}^{\prime} \subset \mathcal{D}^{\prime l} \mid V$ for some open $V \subset \mathbb{R}^{m}$. Let $\mathcal{I}:=0+\mathcal{I}^{\prime} \subset \mathcal{D}^{l, l} \mid U \times V \supset \widetilde{\mathcal{I}}:=0 \pm \mathcal{I}^{\prime}$. For any $f \in H^{0}(U \times V, \widetilde{\mathcal{I}})$, $x=\left(x_{1}, \ldots, x_{r}\right) \in U, y \in V$ we obtain

$$
\frac{\partial f}{\partial x_{i}}\left(x^{0}, y\right)=\lim _{t \rightarrow 0} \frac{f\left(x^{0}+t \cdot e_{i}, y\right)-f\left(x^{0}, y\right)}{t},
$$

which converges in the $C^{\infty}$-topology of $H^{0}\left(V, \mathcal{D}^{\prime l}\right)$. But this implies that $\left(\partial f / \partial x_{i}\right)\left(x^{0},-\right) \in \mathcal{I}^{\prime}$ for any $x^{0} \in U$, because $\mathcal{I}^{\prime}$ is closed and $f\left(x^{\prime},-\right) \in \mathcal{I}^{\prime}$ for any fixed $x^{\prime} \in U$. If $T_{p}$ denotes the mapping which associates to each $C^{\infty}$ function its Taylor series at $p$, we obtain by induction for any $\left(x^{0}, y^{0}\right) \in U \times V$

$$
T_{\left(x^{0}, y^{0}\right)} f=\sum_{\alpha}\left(x-x^{0}\right)^{\alpha} P_{\alpha}\left(y-y^{0}\right) \quad \text { with } \quad P_{\alpha}\left(y-y^{0}\right) \in T_{y^{0}}\left(\mathcal{I}_{y^{0}}^{\prime}\right) .
$$

$T_{y^{0}}\left(\mathcal{I}_{y^{0}}^{\prime}\right)$ is finitely generated, we therefore obtain

$$
T_{\left(x^{0}, y^{0}\right)} f \in T_{\left(x^{0}, y^{0}\right)}\left(\mathcal{I}_{\left(x^{0}, y^{0}\right)}\right)
$$

for each $\left(x^{0}, y^{0}\right) \in U \times V$.

Whitney's spectral theorem $([43], \mathrm{V})$ implies $f \in \overline{\mathcal{I}}=0 \mp \mathcal{I}^{\prime}$. Hence $0 \pm \mathcal{I}^{\prime} \subset 0 \mp \mathcal{I}^{\prime}$. But $0 \mp \mathcal{I}^{\prime} \subset 0 \pm \mathcal{I}^{\prime}$ is obvious. Therefore $0 \pm \mathcal{I}^{\prime}=0 \mp \mathcal{I}^{\prime}$.

Assume now $l=\omega$. Again consider $X=U \subset \mathbb{R}^{r}$ open, $Y=D^{\prime l}=$ $\left(D^{\prime}, \mathcal{D}^{\prime l} / \mathcal{I}^{\prime}\right) \subset \mathbb{R}^{m}$. Let $\mathcal{I}^{\prime \prime}:=\overline{\mathcal{I}^{\prime} \cdot \mathcal{D}^{\infty}}$. The first part gives $0 \pm \mathcal{I}^{\prime \prime}=0 \mp \mathcal{I}^{\prime \prime}$. This implies

$$
\begin{aligned}
0 \pm \mathcal{I}^{\prime}=\left(0 \pm \mathcal{I}^{\prime \prime}\right) \cap \mathcal{D}^{\omega}=\left(0 \mp \mathcal{I}^{\prime \prime}\right) \cap \mathcal{D}^{\omega} & =0 \mp \mathcal{I}^{\prime}, \\
& =0+\mathcal{I}^{\prime}
\end{aligned}
$$


because analytic ideals are always closed (standard). The case $l=\omega^{*}$ follows similarly. In particular, $(\delta)$ is also proved.

Note. $(\gamma)$ is due to K. Reichard ([23], Satz 2.5, c). We shall need even three times mixed spaces. To avoid their introduction we proceed directly as follows: Let $l \in\left\{0, \infty, \omega, \omega^{*}\right\}, X, Y C^{l}$-differentiable spaces, $Z$ a $C^{k}$ differentiable space. We have projections (for $\bullet \in\{\times, \overline{\times}, \underline{\times}\}$ )

$$
\left(\pi_{1}, \pi_{2}, \pi_{3}\right):(X \bullet Y) \bullet Z \rightarrow(X \bullet Y, Z) \rightarrow(X, Y, Z) .
$$

Note. Let $f: X \rightarrow X^{\prime} \in \mathcal{R}^{l}, g: Y \bullet Z \rightarrow Z^{\prime} \in \mathcal{R}^{l+k}$ be differentiable. Then $f, g$ induce in a natural way a differentiable morphism $f \bullet g$ so that the following diagram commutes:

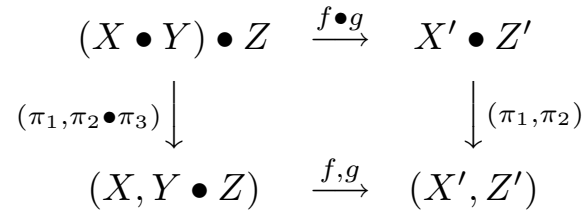

Pr o of (see [29]). We pass to local representatives, for example $F, G$ of $f, g$. Then $F \times G$ is a local representative of $f \bullet g$. This construction does not depend on the choice of the representatives $F, G$ of $f, g$.

For a given reduced $l$-differentiable group (for $l=0$ a topological group; $\left.l \in\left\{0, \infty, \omega, \omega^{*}\right\}\right) G=(G, m, e, i)$ with $m=$ multiplication in $G, e=$ identity, $i=$ inversion ([33]) and for $k \geq l$ we define:

Definition. $G$ is called a transformation group on a closed $k$-differentiable space $X$ by means of a differentiable $f: G \times X \rightarrow X$ if $f \circ(m \times \underline{\text { id }})=$ $f \circ(\mathrm{id} \times f)$, and the composition of the natural mappings $X \hookrightarrow e \underline{\times} X \hookrightarrow$ $G \underline{\times} X \stackrel{f}{\rightarrow} X$ is the identity. $G$ operates effectively if $f_{p}: X \hookrightarrow p \underline{\times} X \hookrightarrow$ $G \underline{\times} X \stackrel{f}{\longrightarrow} X$ is different from the identity for each $p \in G \backslash\{e\}$.

Note. $f_{p}$ is always a diffeomorphism. In situations appearing above $\underline{x}$ is the product $\bar{x}$ in the category of closed spaces according to 2.1 , or it is the product $\times$ in the subcategory of closed, locally finitely generated spaces.

The reader may extend the notion of transformation groups also to nonreduced groups.

$\S 3$. Topological transformation groups on spaces. We show that, in general, topological transformation groups on differentiable spaces are necessarily Lie groups. The proof requires several preparations. Let $l \in$ $\left\{1,2, \ldots, \infty, \omega^{*}\right\}$. Recall that " $X \in \mathcal{R}_{s}^{l}$ " means " $X$ is a standard space", i.e. $X$ is locally of the form $\left(D, \mathcal{D}^{l} / \mathcal{I}\right) \subset \mathbb{R}^{n}$, where $D$ is locally compact 
and $\mathcal{I}$ satisfies $\mathcal{I}=\left(\overline{\mathcal{I} \cdot \mathcal{D}^{l-1}}\right) \cap \mathcal{D}^{l}$, in particular $\mathcal{I}=\overline{\mathcal{I}}, \mathcal{I}=\left(\mathcal{I} \cdot \mathcal{D}^{l-1}\right) \cap \mathcal{D}^{l}$ (see [29], [32]), which is just assumption $\mathrm{A}_{3}$.

Assumption. $G=(G, m, e, i)$ is a topological group which operates as a transformation group on a standard space $X$ by means of a morphism $f: G \times X \rightarrow X$.

With the help of $\mathrm{A}_{3}$ we observe first (see [29]):

Note. $(\alpha)$ For each $p^{*} \in \underline{X}$ there exist open sets

$$
e \in U \subset G, \quad p^{*} \in W_{2}^{*} \subset \underline{X}, \quad W_{0} \subset W_{1} \subset W_{2} \subset \mathbb{R}^{n},
$$

$W_{i}$ convex and relatively compact in $W_{i+1}$, such that: $X \mid W_{2}^{*} \simeq\left(D, \mathcal{D}^{l} / \mathcal{I}\right) \subset$ $W_{2}, p^{*} \simeq p \in D \cap W_{0}, D$ closed in $W_{2}$, the ideal sheaf $\mathcal{I} \subset \mathcal{D}^{l} \mid D$ extends to an ideal sheaf $\mathcal{I} \subset \mathcal{D}^{l} \mid W_{2}$ satisfying $\mathrm{A}_{4}$ and having $D$ as its set of zeros. Moreover, $f\left(U \times\left(W_{1} \cap D\right)\right) \subset D, f \mid U \times\left(W_{1} \cap D\right)$ is generated by a $(0, l)$ differentiable mapping $F: U \times W_{2} \rightarrow \mathbb{R}^{n}$ with $F(e$, id $) \mid W_{2}=$ id, $F\left(U, W_{0}\right) \subset$ $W_{1}, F\left(U, W_{1}\right) \subset W_{2}$.

$(\beta)$ The product $m: G \times G \rightarrow G$ will be denoted (as usual) by ".", and $g^{m}=g \cdot g \ldots g$ denotes the $m$-fold product of $g$.

With these notations, in particular with $p^{*} \in \underline{X}$ fixed, $\operatorname{id}_{i}(x)=x_{i}$, $F=\left(F_{1}, \ldots, F_{n}\right): U \times W_{2} \rightarrow \mathbb{R}^{n}$ we generalize a lemma of H. Cartan:

Lemma 3.1(a). For fixed $q \in \mathbb{N}, g \in G$ with $g, g^{2}, \ldots, g^{q} \in U$, id: $x \rightarrow$ $x \in W_{2}, y_{i}(g, x):=F_{i}(g, x)-x_{i}$ we have

$$
F_{i}\left(g^{q}, \mathrm{id}\right)-\mathrm{id}_{i}-\sum_{j} d_{i j}(q, g, \mathrm{id}) \cdot q \cdot y_{j}(g, \mathrm{id}) \in H^{0}\left(W_{0}, \mathcal{I}\right),
$$

$d_{i j}(q, g, x):=\frac{1}{q} \int_{0}^{1}\left(\delta_{i j}+F_{i j}(g, x+t \cdot y)+\ldots+F_{i j}\left(g^{q-1}, x+t \cdot y\right)\right) d t$.

Then

Proof. Set $G_{i}(g, x):=x_{i}+F_{i}(g, x)+\ldots+F_{i}\left(g^{q-1}, x\right), G_{i j}=G_{i x_{j}}$.

$$
\begin{aligned}
G_{i}(g, x+y)-G_{i}(g, x) & =\sum_{j} y_{j} \int_{0}^{1} G_{i j}(g, x+t y) d t, \\
G_{i}(g, x+y)=G_{i}(g, F(g, x)) & =F_{i}(g, x)+\ldots+F_{i}\left(g^{q}, x\right)-q \cdot H_{i}(q, g, x),
\end{aligned}
$$

where $H_{i}(q, g$, id $) \in H^{0}\left(W_{0}, \mathcal{I}\right)$; since $f \circ(m \times$ id $)=f \circ($ id $\times f)$ implies $F\left(g^{s}, F(g, \text { id })\right)_{i}-F\left(g^{s+1}, \text { id }\right)_{i} \in H^{0}\left(W_{0}, \mathcal{I}\right)$, hence

$$
G_{i}(g, x+y)-G_{i}(g, x)=F_{i}\left(g^{q}, x\right)-x_{i}-q \cdot H_{i}(q, g, x),
$$

therefore

(3) $\quad F_{i}\left(g^{q}, x\right)-x_{i}=q \cdot H_{i}(q, g, x)$ 
$\left.+\sum_{j} \frac{1}{q} \int_{0}^{1}\left(\delta_{i j}+F_{i j}(g, x+t \cdot y)+\ldots+F_{i j}\left(g^{q-1}, x+t \cdot y\right)\right) d t \cdot q \cdot\left(F_{j}(g, x)-x_{j}\right)\right)$.

Lemma 3.1(b). Let in addition $G$ be locally compact. Then for each $p^{*} \in$ $\underline{X}$ there exists a compact neighbourhood $U(e) \subset G$ of $e \in G, W_{i}$ as above, such that whenever $g^{q} \in U, \forall q \in \mathbb{N}$, then even $F(g, \mathrm{id})_{i}-\mathrm{id}_{i} \in H^{0}\left(W_{0}, \mathcal{I}\right)$ for each component.

Proof. In the following we may always assume $U, \varepsilon, e_{i j}$ to be "small enough". $F(e$, id $)=$ id then implies: $F_{i j}(\cdot)=\delta_{i j}+\varepsilon_{i j}(\cdot)$ in $U \times \bar{W}_{0}$, hence $F\left(g^{q}, x\right)-x=q \cdot A \cdot(F(g, x)-x)+q \cdot H(q, g, x)$ (by (3)) where $A=A(q, g, x)=E+\varepsilon(\cdot), \forall x \in \bar{W}_{0}, q \in \mathbb{N}$ ( $E$ the unit matrix). Thus $\exists A^{-1}=E+\delta(\cdot), \forall x \in \bar{W}_{0}, q \in \mathbb{N}$, and so

$$
\frac{1}{q} \cdot A^{-1}\left(F\left(g^{q}, \mathrm{id}\right)-\mathrm{id}\right)-(F(g, \mathrm{id})-\mathrm{id}) \in H^{0}\left(W_{0}, \mathcal{I} \cdot \mathcal{D}^{l-1}\right)^{n}
$$

for fixed $q, g$. In the topology of $H^{0}\left(W_{0}, \mathcal{D}^{l-1}\right)$ we have

$$
\frac{1}{q} \cdot A^{-1}(q, g, \mathrm{id}) \cdot\left(F\left(g^{q}, \mathrm{id}\right)-\mathrm{id}\right) \stackrel{q \rightarrow \infty}{\longrightarrow} 0,
$$

hence $F(g$, id $)$ - id $\in H^{0}\left(W_{0}, \mathcal{I}\right)$ because $\left(\overline{\mathcal{I} \cdot \mathcal{D}^{l-1}}\right) \cap \mathcal{D}^{l}=\mathcal{I}$ (by $\mathrm{A}_{3}$, resp. $\mathrm{A}_{s}$ !).

In particular, $F(g$,id) and id induce the same differentiable mapping $X \mid\left(W_{0} \cap D\right) \rightarrow X([29],[31])$. Hence by $2.1(\beta)$ we obtain

Corollary 3.2. With $U, W_{i}$ as above, and $g^{q} \in U, \forall g \in U, q \in \mathbb{N}$,

$$
f\left|U \times\left(W_{0} \cap D\right): U \underline{\times}\left(X \mid\left(W_{0} \cap D\right)\right) \rightarrow X\right|\left(W_{0} \cap D\right)
$$

is the projection onto the second component of the product.

THEOREM 3.3. Let $G$ be a compact topological transformation group operating on an l-differentiable standard space $X$ by means of $f: G \times X \rightarrow X$ and having $p \in \underline{X}$ as a fixed point (the composition $G \rightarrow G \times \underline{x} \hookrightarrow G \underline{X} X \stackrel{f}{\hookrightarrow} X$ is just the projection $G \rightarrow p)$. Then there exists a chart $(V, \varphi)$ of $X$ with $p \in V$ such that the diagram

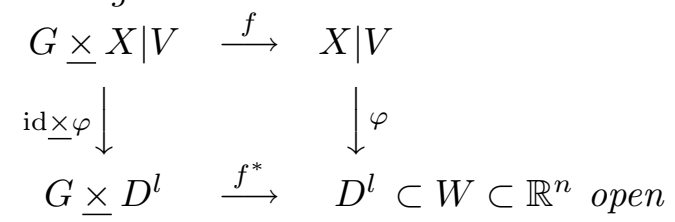

commutes and $f^{*}$ is generated by a $(0, l)$-differentiable mapping $F: G \times W \rightarrow$ $\mathbb{R}^{n}$ with $F(g$, id) linear for each fixed $g \in G$.

Proof. There are arbitrarily small $G$-invariant neighbourhoods of $p \in$ $V \subset \underline{X}$ such that $X \mid V \simeq D^{l}=\left(D, \mathcal{D}^{l} / \mathcal{I}\right) \subset \mathbb{R}^{n}$ with $n=\operatorname{embdim}_{p} X$, 
$p=0 \in \mathbb{R}^{n}, W \subset \mathbb{R}^{n}$ open, $D \subset W$ closed, and $\mathcal{I} \subset \mathcal{D}^{l} \mid D$ extends to an ideal sheaf (also denoted by) $\mathcal{I} \subset \mathcal{D}^{l} \mid W$, which satisfies $\mathrm{A}_{4}$ and has as zero set exactly $D$. In the following we may assume that $V, W$ are sufficiently small neighbourhoods of $p=0 \in \mathbb{R}^{n}$; we identify $X$ with $D^{l}$. Since $G$ is compact, there are finitely many open $U_{i} \subset G$ with the property ( $W$ being sufficiently small!): $\bigcup U_{i}=G$ and $f: U_{i} \times D^{l} \rightarrow D^{l}$ is generated by a $(0, l)$-differentiable mapping $F_{i}: U_{i} \times W \rightarrow \mathbb{R}^{n}$. With the help of a continuous partition of unity on $G$ one finds a $(0, l)$-differentiable mapping $F: G \times W \rightarrow \mathbb{R}^{n}$ generating $f: G \times D^{l} \rightarrow D^{l}$ (using $\mathrm{A}_{4} \Rightarrow \mathrm{A}_{3}$ and [29]).

After these preparations we proceed as follows : For fixed $g \in G$ the morphism $f_{g}: X \rightarrow g \times X \hookrightarrow G \times X \stackrel{f}{\longrightarrow} X$ is a diffeomorphism, the differential $L(g):=d f_{g}(p): \bar{T}_{p} X \rightarrow T_{p} \bar{X}\left(=\mathbb{R}^{n}\right)$ on the tangent spaces is thus a linear isomorphism $([29])$.

AsSERTION 1. $L: G \rightarrow$ Aut $\mathbb{R}^{n}$ is a continuous homomorphism of groups. Therefore $L$ can be considered as a continuous mapping $L: G \times \mathbb{R}^{n} \rightarrow \mathbb{R}^{n}$, which is linear in the second variable.

Proof. $L$ is continuous, because $L(g)=d f_{g}(p)=d_{x} F(g, p)(n=$ $\left.\operatorname{embdim}_{p} X\right)$ depends continuously on $g !\left(d_{x} F\right.$ is the differential of $F$ with respect to the second variable). $L$ is a homomorphism, because $f \circ(\mathrm{id} \times f)=$ $f \circ(m \times$ id $)$ implies for the representative $F$ of $f$ and for fixed $g, h \in G$,

$$
F(g, F(h, \mathrm{id}))-F(g \cdot h, \mathrm{id}) \in H^{0}(W, \mathcal{I})^{n} .
$$

Since $n=\operatorname{embdim}_{p} X, p=$ fixed point, this gives

$$
L(g) \circ L(h)=d_{x} F(g, F(h, p)) \circ d_{x} F(h, p)=d_{x} F(g \cdot h, p)=L(g \cdot h) .
$$

For the next partial result we remark first that $F: G \times W \rightarrow \mathbb{R}^{n}$ above can be considered as a continuous mapping $F: G \rightarrow H^{0}\left(W, \mathcal{D}^{l}\right)^{n}$. Defining $H(g):=L\left(g^{-1}\right) \circ F(g)$ we obtain a continuous mapping $H: G \rightarrow$ $H^{0}\left(W, \mathcal{D}^{l}\right)^{n}$. Integrating with respect to a right invariant normalized Haar measure on $G$, we obtain

$$
R:=\int_{G} H(g) d g \in H^{0}\left(W, \mathcal{D}^{l}\right)^{n} .
$$

Assertion 2. $d R(p): \mathbb{R}^{n} \rightarrow \mathbb{R}^{n}$ is bijective. $R: W \rightarrow R(W)$ is therefore a diffeomorphism of class $C^{l}$ if $W$ is chosen small enough.

Proof. $d_{x} H(g)(p)=E, \forall g \in G$.

Assertion 3. For $a \in G$ we have

$$
\begin{aligned}
L(a) \circ R & =L(a) \circ \int_{G} L\left(g^{-1}\right) \circ F(g) d g=\int_{G} L\left(a g^{-1}\right) \circ F(g) d g \\
& =\int_{G} L\left(h^{-1}\right) \circ F(h \cdot a) d h, \quad \text { where } h=g \cdot a^{-1}
\end{aligned}
$$




$$
\begin{aligned}
& =\int_{G} L\left(h^{-1}\right) \circ(F(h) \circ F(a)+K(h)) d h, \quad K(h) \in H^{0}(W, \mathcal{I})^{n} \\
& =\left(\int_{G} L\left(h^{-1}\right) \circ F(h) d h\right) \circ F(a) \quad \bmod H^{0}(W, \mathcal{I})^{n}
\end{aligned}
$$

because of $\mathrm{A}_{4}$, hence $\mathrm{A}_{3}$. Here we have a continuous mapping $K: G \rightarrow$ $H^{0}(W, \mathcal{I})^{n}$, given for fixed $a \in G$ by

$$
K(h):=F(h \cdot a, \mathrm{id})-F(h, F(a, \mathrm{id})) \in H^{0}(W, \mathcal{I})^{n} .
$$

Thus

$$
L(a) \circ R-R \circ F(a) \in H^{0}(W, \mathcal{I})^{n} .
$$

$R$ induces a diffeomorphism $\varrho: D^{l} \rightarrow D^{* l}$ for some space $D^{* l} \subset R(W) \subset \mathbb{R}^{n}$. By $\mathrm{A}_{3}$ equation $(* *)$ implies ([29]) that $L \circ(\mathrm{id} \times R)$ and $R \circ F$ induce the same morphism. The following diagram, where $\widetilde{L}$ is induced by $L$, is therefore commutative:

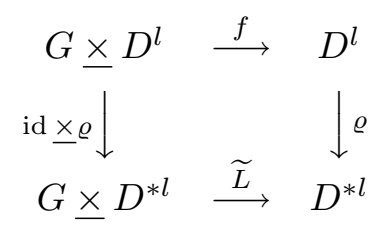

MaIN TheOREm 3.4. Let $G$ be a locally compact topological group, operating effectively as a transformation group on a $C^{l}$-differentiable standard space $X, l \in\left\{1,2, \ldots, \omega, \omega^{*}\right\}$. Assume that there are connected subsets $V_{j} \subset \underline{X}, j=1, \ldots, s$ such that $\bigcup V_{j}=\underline{X}$ and $\operatorname{embdim}_{p} X=$ const., $\forall p \in V_{j}$. Then $G$ is a Lie group (of class $C^{\omega}$ ).

Pr o of. We show that $G$ has no small subgroups. Then $G$ is a Lie group by Montgomery-Zippin [17]. Fix $x_{j} \in V_{j}$ and choose a sufficiently small neighbourghood $U_{j} \subset G$ of $e \in G$ according to 3.1(b). Let $U:=\bigcap U_{i}$, without loss of generality assumed to be compact. We assume, indirectly, that $G$ has small subgroups. Hence in each $U$ there exists a closed subgroup $e \neq G^{*} \subset U . G^{*}$ is compact, and by 3.2 for some neighbourhood $W_{j}\left(x_{j}\right) \subset$ $\underline{X}$ the morphism $G^{*} \underline{\times} X\left|W_{j} \rightarrow G \underline{\times} X\right| W_{j} \stackrel{f}{\longrightarrow} X \mid W_{j}$ is the projection $\forall j$. Therefore there exists a maximal open subset $W \subset \underline{X}$ such that the following composition is just the projection:

$$
G^{*} \times \underline{\times} X|W \rightarrow G \underline{\times} X| W \stackrel{f}{\longrightarrow} X \mid W .
$$

The following assertion will finish the proof of 3.4.

Assertion. $W=\underline{X}$.

We already know that $W \cap V_{j} \neq \emptyset, \forall j$. Choose any $z \in \overline{W \cap V_{j}} \cap V_{j}$ and represent $X$ near $z$ as some $D^{l} \subset \mathbb{R}^{c_{j}}$, where $c_{j}=\operatorname{embdim}_{z} X$. Since $z$ is a 
limit of fixed points $z_{v} \in V_{j} \cap W$, it is also a fixed point. Using 3.3 we may choose the local representation $D^{l}$ in such a way that for each $g \in G^{*}$

$$
f_{g}: D^{l} \rightarrow g \underline{\times} D^{l} \hookrightarrow G^{*} \underline{\times} D^{l} \stackrel{f}{\longrightarrow} D^{l}
$$

is generated by a linear map $L_{g}: \mathbb{R}^{c_{j}} \rightarrow \mathbb{R}^{c_{j}}$. Since $c_{j}=\operatorname{embdim}_{z} D^{l}$ $\forall z \in V_{j} \cap D$, we have

$$
d f_{g}(z)=L_{g}, \quad d f_{g}\left(z_{v}\right)=\text { id } \quad \forall z_{v} \in V_{j} \cap W
$$

( $f$ is a projection there) $\Rightarrow L_{g}=\mathrm{id} \Rightarrow f_{g}$ is the identity $\forall g \in G^{*} \Rightarrow G^{*} \underline{\times}$ $D^{l} \stackrel{f}{\longrightarrow} D^{l}$ is the projection (definition of $\underline{x !}$ ) $\Rightarrow z \in W \cap V_{j} \Rightarrow W \cap V_{j}=\overline{V_{j}}$ because $V_{j}$ is connected $\Rightarrow W=\underline{X}$.

Note. An $\omega^{*}$-differentiable group is a group in the category of $\omega^{*}$ differentiable spaces, which includes the category of $\omega$-differentiable spaces as a full subcategory, as well as the category of complex spaces. So one may say that $G$ is an $\omega^{*}$-differentiable group. But this formulation is not very precise, in particular because it does not necessarily imply that $G$ is a complex Lie group. The same care has to be taken for transformation groups.

$\S 4$. Differentiability of continuous Lie group operations. A Lie group $G$ can be considered as a topological or as a differentiable group (of any class $\left.C^{1}, \ldots, C^{\omega^{*}}\right)$. $G$ may operate on an $l$-differentiable space $X$ as a continuous or a $C^{l}$-differentiable transformation group by means of some $f: G \times X \rightarrow X$, where $G \times X$ is to be taken either as a $(0, l)$-differentiable or an $l$-differentiable space. If $G$ operates on $X C^{l}$-differentiably, then also continuously. The converse also holds in general, as will be proved now. For simplification we restrict our considerations here to the cases $l \in\left\{\infty, \omega^{*}\right\}$.

Assumption. We fix a connected Lie group $G$ with product $m: G \times$ $G \rightarrow G$. In some properly chosen neighbourhood $V^{*}(e) \subset G$ of the unit $e \in G$ we use a normal coordinate system: We consider $V^{*}$ as an open subset $V^{*} \subset \mathbb{R}^{m}$ with $e=0 \in \mathbb{R}^{m}$; and choosing properly some other neighbourhood $V(e) \subset V^{*}$ with $m(V \times V) \subset V^{*}$ we have $m(a, b)=a+b$ whenever $a, b$ are linearly dependent. $V, V^{*}$ may also be assumed to be symmetric, convex and "sufficiently small" for all what follows.

Now let $G$ operate continuously on a given $C^{l}$-differentiable standard space $X, l \in\left\{\infty, \omega^{*}\right\}$, by means of some $(0, l)$-morphism $f: G \underline{\times} X \rightarrow X$. We show that $f$ is $C^{l}$-differentiable. Since $f \circ(m \underline{x i d})=f \circ($ id $\underline{x} f)$ we only have to prove that for each $p \in \underline{X}$ there exist neighbourhoods $U(p) \subset \underline{X}$, $V(e) \subset G$ such that the restriction $f: V(e) \underline{\times} X \mid U(p) \rightarrow X$ is differentiable. 
Therefore fix $p \in \underline{X}$. In some neighbourhood $W_{2}^{*}(p) \subset \underline{X}$ we may consider $X \mid W_{2}^{*}$ as an embedded space

$$
X \mid W_{2}^{*}=D^{l}=\left(D, \mathcal{D}^{l} / \mathcal{I}\right) \subset \mathbb{R}^{n}
$$

with $p=0 \in \mathbb{R}^{n}, W_{2} \subset \mathbb{R}^{n}$ open and $D \subset W_{2}$ relatively closed, and moreover, with $\mathcal{I} \subset \mathcal{D}^{l} \mid D$ being the restriction of a closed ideal $\mathcal{I} \subset \mathcal{D}^{l} \mid W_{2}$ which has $D$ as its set of zeros.

For properly chosen $V^{*}=V^{*}(e) \subset G$ there exist convex neighbourhoods $p=0 \in W=W_{-1} \subset W_{0} \subset W_{1} \subset W_{2} \subset \mathbb{R}^{n}$ such that $f: V^{*} \times X \mid W_{1} \cap D \rightarrow$ $X \mid W_{2}^{*}$ is generated by some $(0, l)$-differentiable mapping $F: V^{*} \times W_{2} \rightarrow \mathbb{R}^{n}$ satisfying $F\left(V^{*}, W_{i}\right) \subset W_{i+1}$ for each $i \in\{-1,0,1\}$ and $F \mid 0 \times W_{2}=\mathrm{id}$. We define

$$
\begin{aligned}
y & :=F(v \cdot r, x) \quad \text { for } v \in V^{*}, x \in W_{2}, 0 \leq r \leq 1, \\
H(v, x) & :=\int_{0}^{1} F(v \cdot t, x) d t \quad \text { for } v \in V^{*}, x \in W_{2} .
\end{aligned}
$$

$H$ is continuous in the $v$-variable, differentiable in the $x$-variable. The differential $d_{x} H$ of $H$ with respect to $x$ exists and is continuous in $v$, differentiable in $x$. We consider $d_{x} H$ as an $(n \times n)$-matrix. Then we have for $v \in V$, $x, y \in W_{0}$

$$
\begin{aligned}
\int_{0}^{1} & d_{x} H(v, x+(y-x) \cdot s) d s \cdot \frac{y-x}{r}=\frac{1}{r}(H(v, y)-H(v, x)) \\
& =\frac{1}{r} \int_{0}^{1}(F(v \cdot t, F(v \cdot r, x))-F(v \cdot t, x)) d t \\
& =\frac{1}{r} \int_{0}^{1}(F(v \cdot t+v \cdot r, x)-F(v \cdot t, x)) d t \quad \bmod 0 \underline{\times} \mathcal{I} \\
& =\frac{1}{r}\left(\int_{r}^{1+r} F(v \cdot t, x) d t-\int_{0}^{1} F(v \cdot t, x) d t\right) \quad \bmod 0 \underline{x} \mathcal{I},
\end{aligned}
$$

hence

$$
\frac{1}{r}(F(v \cdot r, x)-x)=A^{-1} \cdot \frac{1}{r} \cdot K(r, v, x) \quad \bmod 0 \underline{x} \mathcal{I}
$$

Here "mod $0 \times \mathcal{I}$ " means that the identities above hold up to some mapping $G(r, v, x)$, which is continuous in $r, v$, differentiable in $x$ and for fixed $r, v$ is a section in $\mathcal{I}$. This holds because $\mathcal{I}$ is closed and $F(v \cdot t, F(v \cdot r, x))-F(v \cdot t+$ $v \cdot r, x)$ is in $\mathcal{I}$ for fixed $v, t, r$. Next, $A(r, v, x):=\int_{0}^{1} d_{x} H(v, x+(y-x) \cdot s) d s$ is continuous in $r, v$, differentiable in $x$ and as $(n \times n)$-matrix everywhere invertible $\left(d_{x} F(0,-)=\mathrm{id} ; V, W_{2}\right.$ sufficiently small!). Finally, $K$ denotes the 
last difference of integrals and is also continuous in $r, v$ and differentiable in $x$. Substituting $x$ by $F(a, x)$ we obtain

$$
\begin{aligned}
& \text { (**) } \frac{1}{r}(F(v \cdot r, F(a, x))-F(a, x))=A^{*-1} \cdot \frac{1}{r} \cdot K^{*}(r, v, a, x) \quad \bmod 0 \times \underline{I}, \\
& (* * *) \quad \lim _{(r, v, a) \rightarrow\left(0, v^{0}, a^{0}\right)} \frac{1}{r} \cdot K^{*}(r, v, a, \mathrm{id})=F\left(v^{0}, F\left(a^{0}, \mathrm{id}\right)\right)-F\left(a^{0}, \mathrm{id}\right) .
\end{aligned}
$$

The left side of $(* *)$ is considered as a continuous mapping

$$
B: I \times V \times V \rightarrow H^{0}\left(W_{0}, \mathcal{D}^{l}\right)^{n},
$$

where $I:=\{r|0<| r \mid \leq 1\}$. Let $B^{*}$ be the composition of $B$ with $H^{0}\left(W_{0}, \mathcal{D}^{l}\right)^{n} \rightarrow H^{0}\left(W_{0}, \mathcal{D}^{l}\right)^{n} / H^{0}\left(W_{0}, \mathcal{I}\right)^{n}$. $\quad B^{*}$ can be continuously extended onto $\bar{I} \times V \times V$ (by $(* * *))$. With $Q(r, v, a):=m\left(a+v \cdot r, a^{-1}\right)$ one obtains

$$
Q(0, v, a) \equiv 0, \quad Q(r, v, a)=w(r, v, a) \cdot r,
$$

which implies

$$
F(w \cdot r, F(a, x))=F(Q(r, v, a), F(a, x))=F(a+v \cdot r, x) \quad \bmod 0 \underline{x} \mathcal{I},
$$

and

$$
\begin{aligned}
(* * * *) & \frac{1}{r} & (F(a+v \cdot r, x)-F(a, x)) & \\
& =\frac{1}{r} \cdot(F(w \cdot r, F(a, x))-F(a, x)) & & \bmod 0 \times \underline{\mathcal{I}} \\
& =A^{*-1} \cdot \frac{1}{r} \cdot K^{*}(r, w, a, x) & & \bmod 0 \underline{\times} \mathcal{I} .
\end{aligned}
$$

We consider $F$ as a continuous mapping $F: V \rightarrow H^{0}\left(W_{0}, \mathcal{D}^{l}\right)^{n}$. Let $F^{*}$ denote the composition of $F$ with $H^{0}\left(W_{0}, \mathcal{D}^{l}\right)^{n} \rightarrow H^{0}\left(W_{0}, \mathcal{D}^{l}\right)^{n} / H^{0}\left(W_{0}, \mathcal{I}\right)^{n}$. (****) implies that $F^{*}: V \rightarrow H^{0}\left(W_{0}, \mathcal{D}^{l}\right)^{n} / H^{0}\left(W_{0}, \mathcal{I}\right)^{n}$ is continuously differentiable. Consider the differential $d_{g} F^{*}$ as an $(n \times m)$-matrix, the coefficients being continuous mappings $V \rightarrow H^{0}\left(W_{0}, \mathcal{D}^{l}\right) / H^{0}\left(W_{0}, \mathcal{I}\right)$. For each fixed $a \in V$ we have

$$
\begin{gathered}
d_{g} F^{*}(a) \in\left(H^{0}\left(W_{0}, \mathcal{D}^{l}\right) / H^{0}\left(W_{0}, \mathcal{I}\right)\right)^{n \cdot m}, \\
d_{g} F^{*}(a) \cdot v \in H^{0}\left(W_{0}, \mathcal{D}^{l}\right)^{n} / H^{0}\left(W_{0}, \mathcal{I}\right)^{n} \quad \forall v \in \mathbb{R}^{m} .
\end{gathered}
$$

$F$ generates $f$, and so for fixed $a \in V$ a morphism $D^{l}\left|W \rightarrow D^{l}\right| W_{0}$, and by "substitution with $F^{*}(a)$ " also a mapping $H^{0}\left(W_{0}, \mathcal{D}^{l}\right) / H^{0}\left(W_{0}, \mathcal{I}\right) \rightarrow$ $H^{0}\left(W, \mathcal{D}^{l}\right) / H^{0}(W, \mathcal{I})$. In particular, we have $\left(d_{g} F^{*}(0) \cdot v\right) \circ F^{*}(a) \in$ $\left(H^{0}\left(W, \mathcal{D}^{l}\right) / H^{0}(W, \mathcal{I})\right)^{n}$; furthermore, the resulting mapping $V \rightarrow$ $\left(H^{0}\left(W, \mathcal{D}^{l}\right) / H^{0}(W, \mathcal{I})\right)^{n}$ is continuous. With $a=v \cdot t$ we obtain

$\frac{1}{r}(F(v \cdot t+v \cdot r, x)-F(v \cdot t, x))=\frac{1}{r}(F(v \cdot r, F(v \cdot t, x))-F(v \cdot t, x)) \quad \bmod 0 \times \underline{\mathcal{I}}$. 
Letting $r \rightarrow 0$ we therefore obtain in $W$ for fixed $v, t$

$$
d_{g} F^{*}(v \cdot t) \cdot v=\left(d_{g} F^{*}(0) \cdot v\right) \circ F^{*}(v \cdot t) .
$$

With $B \in H^{0}\left(W_{0}, \mathcal{D}^{l}\right)^{n \cdot m}$ as a representative of $d_{g} F^{*}(0)$ and with $A(v, x):=$ $B(x) \cdot v$ we obtain by integration

$$
F(v \cdot t, x)-x=\int_{0}^{t} A(v, F(v \cdot t, x)) d t \quad \bmod 0 \underline{\times} \mathcal{I} .
$$

On the other hand, there exists a solution $G_{t}(t, v, x)=A(v, G(t, v, x))$ with $G(0, v, x) \equiv x$, which is differentiable in all variables. Therefore, as in the usual theory of ordinary differential equations, $F$ and $G$ are $\bmod 0 \pm \mathcal{I}$ fixed points of the same contracting operator on the Fréchet space $\left(\bar{H}^{0}\left(W, \mathcal{D}^{l}\right) / H^{0}(W, \mathcal{I})\right)^{n}$. This implies

$$
G(t, v, x) \equiv F(v \cdot t, x) \quad \bmod 0 \underline{x} \mathcal{I} .
$$

But this means that $G(1,-)$ and $F$ generate the same morphism $f$ of spaces $([29])$. Therefore $f$ is $l$-differentiable in some neighbourhood of $(e, p) \in$ $G \times \underline{X}$. So we have proved for $l \in\left\{\infty, \omega, \omega^{*}\right\}$ :

THEOREM 4.1. Let $G$ be a Lie group of class $C^{l}$ operating continuously on a $C^{l}$-differentiable standard space $X$. Then $G$ operates l-differentiably.

N ot e. Locally compact $\omega$-, $\omega^{*}$-differentiable spaces are always standard spaces.

COROllary 4.2. Let $G$ be a locally compact, connected l-differentiable group, $l \in\left\{\infty, \omega, \omega^{*}\right\}$. Then $\operatorname{red} G$ is a Lie group (in general of class $C^{\omega}$ only, see the note at the end of Section 3$)$.

Proof. Let $G$ be already reduced. By means of the multiplication $m: G \times G \rightarrow G$ the group $G$ operates on $G$. We consider $G$ as a continuous transformation group $G_{1}$ which operates effectively on the differentiable space $G$ (which is reduced and locally compact, hence even a standard space). Now $\operatorname{embdim}_{e} G=\operatorname{embdim}_{p} G, \forall p \in G$. By 3.4 the topological group $G_{1}$ arises from a Lie group $G_{2}$, and with respect to this structure the operation $m: G_{2} \times G \rightarrow G$ is differentiable by 4.1. In particular, $m: G_{2} \times \underline{x} e \rightarrow G_{2} \times G \rightarrow G$ is differentiable, even a diffeomorphism. Therefore $G_{2}=G$.

Remarks. $(\alpha)$ Continuous group operations $G \underline{x} X \rightarrow X$ on a space $X$ induce a foliation $\mathcal{F}_{G}$ on $X$ and the leaf space $X / \mathcal{F}_{G}$ as quotient space. Under weak assumptions $G$ operates already differentiably on $X$ as a Lie group (3.4 and 4.1), and moreover the leaf space is a differentiable space, being a quotient space ([22]-[24]), $G$ operating properly). Note that in general the quotient space is a differentiable space with singularities, even if the original 
space $X$ is a differentiable manifold. However, certain other properties of a space $X$ are maintained in the quotient $X / \mathcal{F}_{G}([23])$. Moreover, certain more general foliations $\mathcal{F}$ on $X$ are essentially stable iff the quotient $X / \mathcal{F}$ is a differentiable space $([4]),[14],[25],[35])$; [24] describes how "product decompositions" of quotient-space-germs $\left(X / \mathcal{F}_{G}\right)_{p}$ are reflected in product decompositions of the group $G$. Differentiable spaces thus constitute a good category in this context.

( $\beta$ ) Differentiable spaces of different classes were first introduced in [3], [28], [29]. The special case of reduced differentiable spaces was also introduced and discussed in the following years by other authors under different names, for example: subeuclidean spaces in [1], [2], [38], [39]; $D_{0}$ differential spaces $\left(=C^{\infty}\right.$-reduced differentiable spaces; the more general differential spaces are essentially what is known under the more general name of ringed spaces with derivations) in [26], [27]; and others. Their results are essentially contained in our framework, for example [1], [2], [5], [18], [26], [27] in [29]-[31]; [13] in [12], [29]; [15] in [31]. [6] for example gives a correct and extended version of incorrect constructions in [38] in terms of differentiable spaces.

$(\gamma)$ For $l$-differentiable standard, resp. complex spaces $X, Y$ we topologize ([10], [17]) $\operatorname{Hom}(X, Y):=\{g \mid g: X \rightarrow Y$ is differentiable $\}$, in particular

$$
\operatorname{Hom}(X, X) \supset \operatorname{Aut}(X):=\{g \mid g: X \rightarrow X \text { is a diffeomorphism }\}
$$

in such a way that we obtain a $(0, l)$-differentiable mapping $f: \operatorname{Hom}(X, Y) \underline{\times}$ $X \rightarrow Y$ with

(*) $\quad g=f_{g}: X \hookrightarrow g \underline{x} X \hookrightarrow \operatorname{Hom}(X, Y) \underline{\times} X \stackrel{f}{\longrightarrow} Y \quad \forall g \in \operatorname{Hom}(X, Y)$.

The discrete topology on $\operatorname{Hom}(X, Y)$ satisfies this condition. More generally, each topology satisfying this condition has the following property: For each $U \subset \underline{X}$ open, relatively compact, $V \subset \underline{Y}$ open we have:

$\operatorname{Hom}(X, Y) \mid(U, V):=\{g \in \operatorname{Hom}(X, Y) \mid g(\bar{U}) \subset V\} \subset \operatorname{Hom}(X, Y)$ open.

Moreover, for each $h \in H^{0}(V, \underline{\underline{Y}})$ the mapping

$$
h^{*}: \operatorname{Hom}(X, Y) \mid(U, V) \rightarrow H^{0}(\bar{U}, \underline{\underline{X}})
$$

given by $g \rightarrow \underline{g}(h) \in H^{0}(U, \underline{\underline{X}})$ is continuous. Here $H^{0}(U, \underline{\underline{X}})$ carries a natural topology: For $C^{\infty}$-differentiable standard spaces it is described in [29], for complex spaces (which constitute a full subcategory in all $\omega^{*}$-differentiable spaces) for example in [7]. In these cases we topologize $\operatorname{Hom}(X, Y)$ with the smallest topology satisfying the above mentioned properties. Then one may obtain a $(0, l)$-differentiable morphism $f: \operatorname{Hom}(X, Y) \times X \rightarrow Y$ satisfying $(*)$. Moreover, for each topological space $M$ and each $(0, l)$-differentiable $h: M \times X \rightarrow Y$ there exists exactly one continuous $H: M \rightarrow \operatorname{Hom}(X, Y)$ 
satisfying

$$
h=f \circ(H \times \mathrm{id}) .
$$

This characterizes the topology of $\operatorname{Hom}(X, Y)$ again.

The natural mapping $\operatorname{Hom}(X, Y) \times \operatorname{Hom}(Y, Z) \rightarrow \operatorname{Hom}(X, Z)$ is continuous: One has natural $(0, l)$-differentiable morphisms $g_{2}: \operatorname{Hom}(Y, Z) \underline{X} Y \rightarrow$ $Z, g_{1}: \operatorname{Hom}(X, Y) \times X \rightarrow Y$, hence an induced morphism

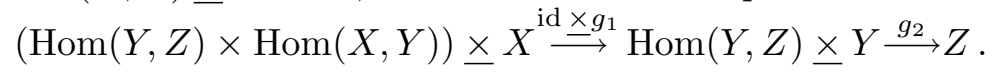

In particular, in the above mentioned cases Aut $X$ is a semitopological group in the sense of [8], hence a topological group (by [8], since Aut $X$, just as $X$, has countable topology and is metrically complete). Aut $X$ therefore is a topological transformation group on $X$. Connected, locally compact subgroups of Aut $X$ are therefore Lie groups operating differentiably on $X$. For example, the subgroup of isometries on $X$, if $X$ carries a Riemannian metric and satisfies appropriate weak properties, will be a Lie group ([16], p. 83). Concerning more generally infinitesimal transformation groups on differentiable spaces see also [16], [34], [37].

\section{References}

[1] N. Aronszajn, Subcartesian and subriemannian spaces. Notices Amer. Math. Soc. 14, (1967), 111.

[2] N. Aronszajn and P. Szeptycki, Subcartesian spaces, J. Differential Geom. 15 (1980), 393-416.

[3] H. Behnke, K. Spallek et al., Zur lokalen Theorie in der Funktionentheorie mehrerer komplexer Veränderlicher; in particular K. Spallek, Differenzierbare Räume, in: Forschungsergebnisse aus dem 1. Math. Inst. der Univ. Münster, Westdeutscher Verlag, Köln und Opladen 1966.

[4] R. Bekemeier, Holmann-Blätterungen im differenzierbaren Fall (auf differenzierbaren Räumen), Diplomarbeit, Universität Bochum, 1988.

[5] A. Breuer and C. D. Marshall, Banachian differentiable spaces, Math. Ann. 237 (1978), 105-120.

[6] D. Gottowik, Zweiter Tangentialraum und kovariante Ableitungen auf differenzierbaren Räumen, Diplomarbeit, Universität Bochum, 1987.

[7] H. Grauert, Ein Theorem der analytischen Garbentheorie, Publ. Math. 5 (1960), 232-292.

[8] T. Husain, Introduction to Topological Groups, Saunders 1966.

[9] M. Jurchescu, Espaces mixtes, in: Lecture Notes in Math. 1014, Springer, 1983, $37-57$.

[10] W. Kaup, Infinitesimale Transformationsgruppen komplexer Räume, Math. Ann. 160 (1965), 72-92.

[11] H. Kerner, Über die Automorphismengruppe komplexer Räume, Arch. Math. (Basel) 11 (1960), 282-288.

[12] N. Kiesow, Einbettung von Räumen in Mannigfaltigkeiten minimaler Dimension, Dissertation, Universität Bochum, 1979. 
[13] A. Kowalczyk, Whitney's and Nash's embedding theorems for differential spaces, Bull. Acad. Polon. Sci. Sér. Sci. Math. Astronom. Phys. 28 (1980), 385-390.

[14] F. Leymann, Blätterungen von Räumen mit Singularitäten, Dissertation, Universität Bochum, 1987.

[15] C. D. Marshall, Calculus on subcartesian spaces, J. Differential Geom. 10 (1980), 551-573.

[16] H. Meier, Anwendungen der Theorie der lokal integrablen Vektorfelder auf Räumen mit Singularitäten, Dissertation, Universität Bochum, 1986.

[17] D. Montgomery and L. Zippin, Topological Transformation Groups, Interscience Publ., 1966.

[18] D. Motreanu, Embeddings of $C^{\infty}$-subcartesian spaces, An. Stiinţ. Univ."Al. I. Cuza" Iaşi 25 (1979), 65-70.

[19] Z. Pasternak-Winiarski, Group differential structures and their fundamental properties, thesis, Inst. Math., Techn. Univ. Warsaw, 1981 (in Polish).

[20] -, Differential groups of class $\mathcal{D}_{0}$, Abh. Akad. Wiss. DDR, Abt. Math. Naturwiss. Technik 2N (1984), 173-176.

[21] -, Differential groups of class $\mathcal{D}_{0}$ and standard charts, Demonstratio Math. 16 (2) (1983), 503-517.

[22] K. Reichard, Quotienten differenzierbarer Räume nach eigentlich diskontinuierlichen Gruppen, Math. Z. (1976), 281-283.

[23] —, Quotienten analytischer und differenzierbarer Räume nach Transformationsgruppen, Habilitation, Universität Bochum, 1978.

[24] K. Reichard and K. Spallek, Productsingularities and quotients, in: Holomorphic Dynamics, Proc. Mexico, Lecture Notes in Math. 1345, Springer, 1986, 256-270.

[25] U. Schneider-Dunio, Stabilität transversal differenzierbarer Relationensysteme auf allgemeinen Räumen, Diplomarbeit, Universität Bochum, 1988.

[26] R. Sikorski, Abstract covariant derivative, Colloq. Math. 18 (1967), 251-272.

[27] —, Differential modules, ibid. 24 (1971), 45-79.

[28] K. S pallek, Differenzierbare und holomorphe Funktionen auf analytischen Mengen, Math. Ann. 161 (1965), 143-162.

[29] —, Differenzierbare Räume, ibid. 180 (1969), 269-296.

[30] —, Glättung differenzierbarer Räume, ibid. 186 (1970), 233-248.

[31] —, Differential forms on differentiable spaces, I, II, Rend. Mat. (2) 4 (1971), 231258 , and 5 (1972), 375-389.

[32] —, Beispiele zur lokalen Theorie der differenzierbaren Räume, Math. Ann. 195 (1972), 332-347.

[33] -, Zur Klassifikation differenzierbarer Gruppen, Manuscripta Math. 11 (1974), $345-357$.

[34] -, Geometrische Bedingungen für die Integrabilität von Vektorfeldern auf Teilmengen des $\mathbb{R}^{n}$, ibid. 25 (1978), 147-160.

[35] -, Foliations on singularities, in: Complex Analysis and Applications, Proc. Varna 1985, Bulgar. Acad. Sci., Sofia 1986, 643-657.

[36] —, Differentiable groups and Whitney spaces, Serdica 16 (1990), 166-175.

[37] - Fortsetzung von Blätterungen und Integration beliebiger Verteilungen, in: Complex Analysis, Seventh Romannian-Finnish Seminar, Bucarest 1989, Rev. Roumaine Math. Pures Appl. (1991/92), to appear.

[38] P. Szeptycki, Vector bundles on subcartesian spaces, Ann. Polon. Math. 42 (1983), $350-368$.

[39] —, Subcartesian spaces, preprint, 1980. 
[40] M. Teufel, Differenzierbare Strukturen und Jetbündel auf Räumen mit Singularitäten, Dissertation, Universität Bochum, 1979.

[41] F. Treves, Topological Vector Spaces, Distributions and Kernels, Academic Press, 1967.

[42] H. Whitney, Analytic extensions of differentiable functions defined in closed sets, Trans. Amer. Math. Soc. 36 (1934), 63-89.

[43] H. Whitney, On ideals of differentiable functions, Amer. J. Math. 70 (1948), 635658.

Added in proof:

[44] W. Sasin and K. Spallek, Gluing of differentiable spaces and applications, Math. Ann. (1991), to appear.

MATHEMATISCHES INSTITUT

RUHR-UNIVERSITÄT BOCHUM

UNIVERSITÄTSSTR. 150

W-4630 BOCHUM, GERMANY

Reçu par la Rédaction le 12.9.1990 